Unstable crack motion is predictable

F. Abraham

August 23, 2004

Journal of the Mechanics and Physics of Solids 
This document was prepared as an account of work sponsored by an agency of the United States Government. Neither the United States Government nor the University of California nor any of their employees, makes any warranty, express or implied, or assumes any legal liability or responsibility for the accuracy, completeness, or usefulness of any information, apparatus, product, or process disclosed, or represents that its use would not infringe privately owned rights. Reference herein to any specific commercial product, process, or service by trade name, trademark, manufacturer, or otherwise, does not necessarily constitute or imply its endorsement, recommendation, or favoring by the United States Government or the University of California. The views and opinions of authors expressed herein do not necessarily state or reflect those of the United States Government or the University of California, and shall not be used for advertising or product endorsement purposes.

This work was performed under the auspices of the U.S. Department of Energy by the University of California, Lawrence Livermore National Laboratory under Contract No. W-7405-Eng-48. 


\title{
Unstable crack motion is predictable
}

\author{
Farid F. Abraham \\ Lawrence Livermore National Laboratory, Livermore CA 94550 \\ The University of Georgia, Athens GA 30602*
}

\begin{abstract}
Yoffe's linear theory of dynamic brittle fracture suggests that crack motion will be unstable beyond $\sim 70 \%$ of the Rayleigh speed, a prediction that is not supported by experiment. We show by atomistic simulations that hyperelasticity, the elasticity of large strains, plays a governing role in the instability dynamics of brittle fracture. A simple scaling model based on an effective elastic modulus, coupled with Yoffe's solution, gives successful predictions for the onset speed of the crack instability.
\end{abstract}

Keywords: Dynamic fracture, instability, materials failure, hyperelasticity, atomistic simulation, molecular dynamics, Yoffe

*Graham-Purdue Visiting Professor of Physics

One may ask, why is it that scaling laws are of such distinguished importance? The answer is that scaling laws never appear by accident. G.I. Barenblatt, Scaling (Cambridge University Press, 2003)

In 1951, Yoffe $^{1}$ made the physically intuitive suggestion that mode I crack growth occurs in the direction of maximum asymptotic hoop stress and found the crack speed for the onset for branching to be about $70 \%$ of the Rayleigh wave speed $c_{R} \cdot{ }^{2,3}$ This high speed is rarely observed in experiment. ${ }^{4,5}$ An obvious shortcoming in Yoffe's analysis is the assumption of a constant linear elastic response for all deformations.

We show by atomistic simulation that hyperelasticity, the elasticity of large strains, plays a governing role in the instability dynamics of brittle fracture: i.e., the hyperelasticity around the crack tip significantly influences the critical speed at which a 
crack deviates from forward motion. Our simulation model is based on a generalized biharmonic potential composed of two spring constants, one associated with small deformations $\left(k_{1}, r \leq r_{o n}\right.$. ) and the other associated with large deformations $\left(k_{2}, r>r_{o n}\right)$. This is shown in Figure 1(a). This serves as a useful model, allowing us to investigate the generic effects of hyperelasticity by changing the relative magnitude ( $\left.a=k_{2} / k_{1}\right)$ and transition distance $\left(r_{\text {on }}\right)$ of the potential. This potential has been used to investigate the dynamics of cracks constrained to remain straight. ${ }^{6}$

A simple proposal for on an effective elastic modulus, coupled with Yoffe's solution, gives successful predictions for the onset speed of the crack instability in nonlinear materials. We conclude that Yoffe's picture of the dynamic instability in brittle fracture is valid.

\section{Modelling}

We consider the propagation of a crack in a two-dimensional crystal geometry shown in Figure 1(b). The slab lengths are given by $l_{x}$ and $l_{y}$, respectively. The crack propagates in the $\mathrm{x}$ direction, and its extension is denoted by $a$. The slab is loaded in mode I in the $\mathrm{y}$ direction with a constant strain rate equal to 0.00001 (all quantities given are in dimensionless units). A slit of length $a=200$ is cut midway through the slab as an initial, atomically sharp crack. The crack initially propagates in a triangular hexagonal lattice with nearest neighbour distance $r_{0}=2^{\frac{1}{6}} \approx 1.12246$ along a cleavage plane with lowest surface fracture energy (Figure 1(c)). We assume that atomic bonds snap at a critical atomic snapping distance $r_{\text {break }}$. The slab is initialized at zero temperature.

We adopt a biharmonic, interatomic potential composed of two spring constants $k_{1}=72 / \sqrt[3]{3} \approx 57.14$ and $k_{2}=a k_{1}, a=0.5625,0.81,1.0,1.5$ and 2.0. The spring constant $k_{1}$ is associated with displacements from the equilibrium distance $r_{0}$, and the second spring constant $k_{2}$ is associated with large bond stretching for $r>r_{o n}$. For each 
$k_{2}$, the values for $r_{\text {on }}$ are taken to be 1.1257, 1.1290, 1.1360, 1.1375, 1.1449 and 1.1550, respectively. There are three distinct wave velocities in elastic solid: the longitudinal wave speed $c_{l}=\sqrt{\frac{3 \mu}{\rho}}$, the shear wave speed $c_{s}=\sqrt{\frac{\mu}{\rho}}$ and the Rayleigh wave speed $c_{R} \approx 0.9225 c_{s}$, with the density $\rho=2 / \sqrt{3} / \sqrt[3]{2} \approx 0.9165$ for atomic mass $m=1$. For small deformation, the shear modulus $\mu=24.8$.

\section{Results}

Figure 2 shows a comparison of crack speed histories for the biharmonic solid with different $k_{2} / k_{1}$ and a particular transition distance $r_{\text {on }}=1.136$ expressed as $\varepsilon_{0}=\left(r_{\text {on }} / r_{0}\right)-1$ $=0.012$. The histories are similar for $\varepsilon_{0}=0.003,0.006,0.014,0.020$ and 0.029 , corresponding to the respective $r_{\text {on }}$. The dynamic crack instabilities for the various $a=$ $k_{2} / k_{1}$ are associated with the precipitous drops in crack speed, as indicated by the arrows, and are a consequence of the crack deviating from straight line motion (see Figure 3). The crack speed at the onset of erratic motion is defined as the instability speed. Figure 4 presents a log-log plot of the instability speed as a function of $a=k_{2} / k_{1}$ for various $\varepsilon_{0}=\left(r_{o n} / r_{0}\right)-1$. For each $\varepsilon_{0}$, the dependence is essentially linear, the slope approaching one-half for $\varepsilon_{0}$ tending to zero. This trend is required since $k_{2} / k_{1}=1$ for $\varepsilon_{0}$ $=0$ and the solid is strictly harmonic with a spring constant equal to $k_{2}$. Therefore, the instability speed will have a trivial square-root dependence on the harmonic spring constant $k_{2}$ when normalized by $k_{1}$. The other limit is $r_{\text {on }}=r_{\text {break }}\left(\varepsilon_{0}=0.04235\right)$. In this limit, the biharmonic potential is simply the harmonic potential with spring constant $k_{1}$ and with slope equal to zero.

Figure 5 defines, graphically, our choice for an effective harmonic spring constant $k_{\mathrm{eff}}$ of the biharmonic potential. It is simply the slope of the vector sum of the maximum piecewise harmonic forces defined by the biharmonic potential as shown in Figure 5; i.e., 


$$
k_{\mathrm{eff}}=k_{1}\left[\left(r_{\text {on }}-r_{0}\right) /\left(r_{\text {break }}-r_{0}\right)\right]+k_{2}\left[\left(r_{\text {break }}-r_{\text {on }}\right) /\left(r_{\text {break }}-r_{0}\right)\right] \text {. }
$$

By plotting in Figure 6 the instability speed as a function of $a_{\mathrm{eff}}=k_{\mathrm{eff}} / k_{1}$, we see a remarkable collapse of the data from Figure 4 onto a common straight line with slope equal to one-half. Hence, for determining the instability speed of a dynamic brittle crack, this finding allows one to model the biharmonic material as a harmonic solid with the effective spring constant just described.

We apply this concept of an effective spring constant to a continuous interatomic potential: in particular, to the Lennard-Jones 12:6 potential. Our construction of $a_{\mathrm{eff}}=$ $k_{\text {eff }} / k_{1}$ is shown in Figure 7 , and it should be self-explanatory. Consistent with our geometrical construction of $k_{\text {eff }}$ for the biharmonic potential, we adopt the slope for the line passing through the LJ force curve at its equilibrium and its maximum as the effective spring constant. We have defined the spring constant with a L-J subscript for sake of clarity. It is a big jump of faith to apply this simple construction to a continuous potential, but the prediction is quite sensible. In Figure 6, we note that it predicts an instability speed of $\sim 0.42$, not much different from speed of 0.36 observed in computer simulations. $^{8}$

For a simple harmonic solid, the instability speed is 0.73 . For a nonharmonic solid, the instability speed is $0.73 \sqrt{ } k_{\text {eff }} / k_{1}$.

\section{Discussion}

Recent atomistic fracture simulations of a harmonic solid have shown remarkable agreement between the virial hoop stress of statistical mechanics and the asymptotic solution of continuum mechanics. ${ }^{9}$ In that study, the atomic crack was constrained to propagate along a straight path so that such a comparison could be made for crack speeds beyond the instability speed. The main result of that study was that the hoop 
stress near a crack tip using continuum theory is reproduced well in the atomistic simulations. Most important, the Yoffe prediction that the hoop stress field becomes bimodal above 73 percent of the Rayleigh speed is found in MD simulation results as well. This is shown in Figure 8.

In Figure 4, we note that the instability speed for the strictly harmonic solid $(\alpha=1)$ is equal to 73 percent of the Rayleigh speed, in agreement with simulation and the Yoffe prediction. We conclude that Yoffe's picture of the dynamic instability in brittle fracture is valid. It is only necessary to replace the elastic modulus for small deformation with an effective elastic modulus described in this study, giving successful predictions for the onset speed of the crack instability for nonlinear materials. The challenge is to demonstrate this conclusion by a mathematical analysis based on a piecewise linear elasticity analysis. Finally, our finding suggests that any theory that is based strictly on the local elastic properties in the neighbourhood of the crack tip would be incomplete.

\section{References}

1. Yoffe, E. H. The moving Griffith crack. Phil. Mag. 42, 739-750 (1951).

2. Broberg, B. Cracks and Fracture (Academic Press, San Diego, 1999).

3. Freund, L.B. Dynamic Fracture Mechanics (Cambridge University Press, Cambridge, UK, 1998).

4. Fineberg, J. Gross, S.P., Marder, M., and Swinney, H.L. Instability in dynamic fracture. Phys. Rev. Lett. 67, 141-144 (1991).

5. Ravi-Chandar, K. Dynamic fracture of nominally brittle materials. Int. J. of Fract. 90, 83-102 (1998).

6. Buehler M., Abraham F.F., Gao H. Hyperelasticity governs dynamic fracture at a critical length scale, Nature 426, 141-146 (2003).

7. Abraham F.F., Brodbeck D., Rafey R.A., and Rudge W.E. Instability dynamics of fracture: A computer simulation investigation. Phys. Rev. Lett. 73, 272-275 (1994). 
8. Abraham, F.F., Brodbeck, D., Rudge, W.E., Xu, X. A molecular-dynamics investigation of rapid fracture mechanics. J. Mech. Phys. Solids 45, 1595-1619 (1997).

9. Buehler M., Abraham F.F., Gao H. Stress and energy flow field near a rapidly propagating mode I crack. SIAM Multiscale Modeling and Simulation, to appear

\section{Figure Captions}

Figure 1. (a) The biharmonic potential is composed of two spring constants, one associated with small deformations $\left(k_{1}, r \leq r_{o n}\right.$.) and the other associated with large deformations $\left(k_{2}, r>r_{\text {on }}\right)$. (b) The two-dimensional simulation geometry is shown, the slab size being given by $l_{x}, l_{y}$ and the crack extension by $a$. (c) The crack initially propagates in a triangular hexagonal lattice with nearest neighbour distance $r_{0}=2^{\frac{1}{6}}$ along a cleavage plane with lowest surface fracture energy (denoted by dotted line).

Figure 2. A comparison of crack speed histories is shown for the biharmonic solid with different $k_{2} / k_{1}$ and a particular transition distance $r_{\text {on }}=1.136$ expressed as $\varepsilon_{0}=\left(r_{\text {on }} / r_{0}\right)-$ $1=0.012$. The dynamic crack instabilities for the various $a=k_{2} / k_{1}$ are indicated by the arrows.

Figure 3. A picture of a crack is shown at a significant time beyond the onset of the instability.

Figure 4. A log-log plot of the instability speed as a function of $a=k_{2} / k_{1}$ is presented for various $\varepsilon_{0}=\left(r_{\text {on }} / r_{0}\right)-1$.

Figure 5. The effective harmonic spring constant $k_{\text {eff }}$ is defined graphically for the biharmonic potential.

Figure 6. The instability speed is presented as a function of $a_{\mathrm{eff}}=k_{\mathrm{eff}} / k_{1}$.

Figure 7. The construction of $a_{\mathrm{eff}}=k_{\mathrm{eff}} / k_{1}$ is shown. 
Figure 8. Recent atomistic fracture simulations of a harmonic solid have shown remarkable agreement between the virial hoop stress of statistical mechanics (dots) and the asymptotic solution of continuum mechanics (blue lines). 
Figure 1

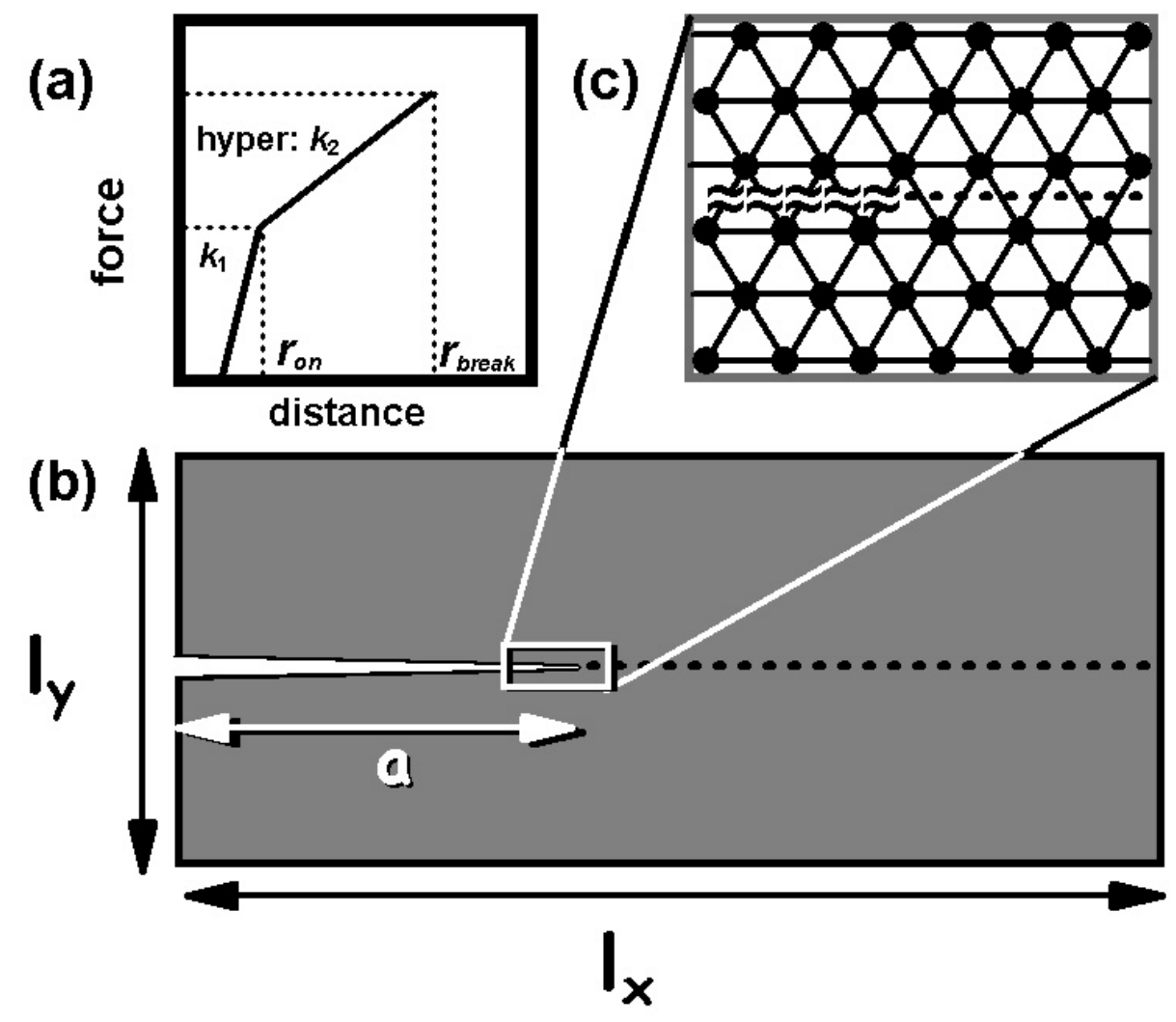


Figure 2

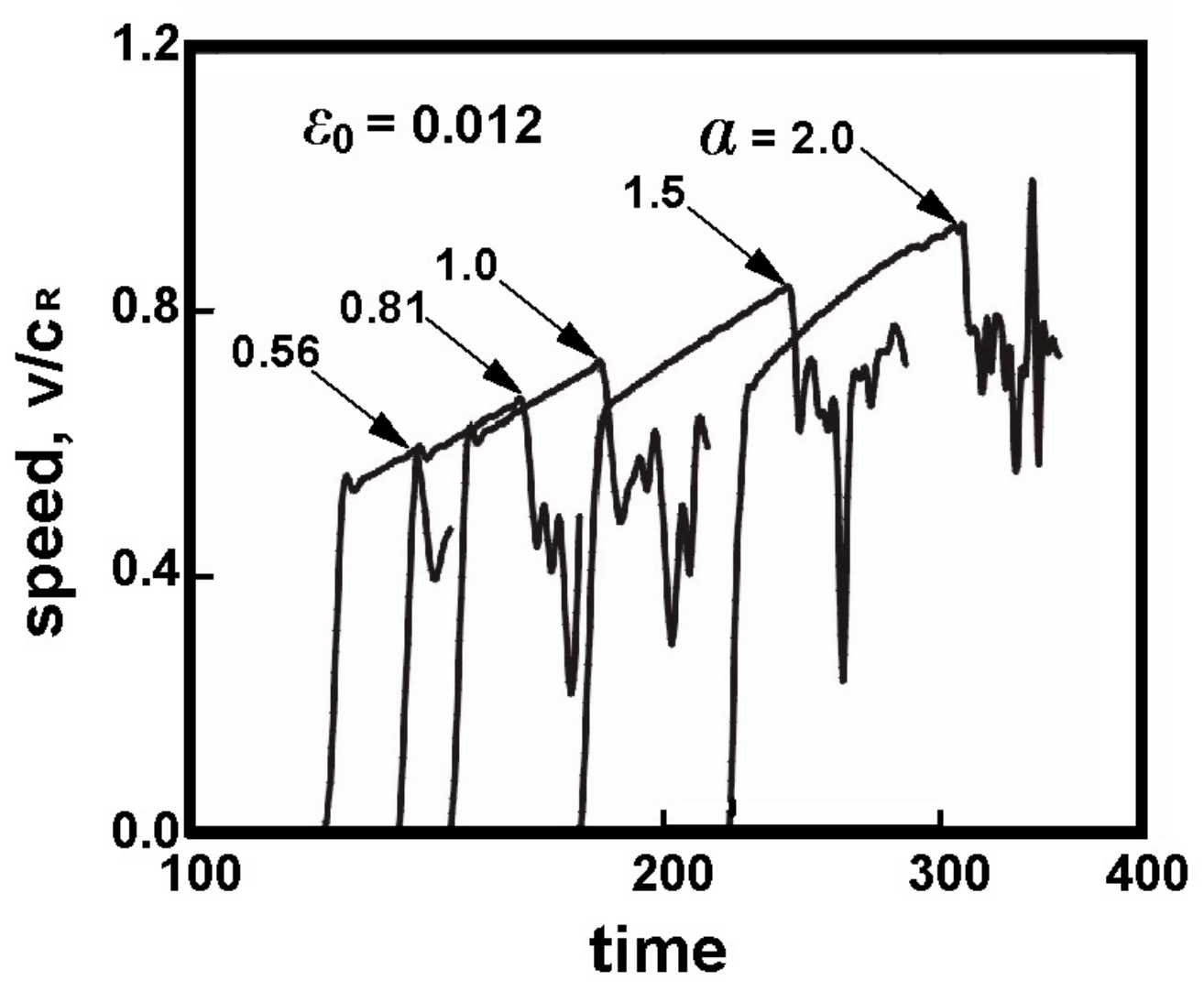


Figure 3

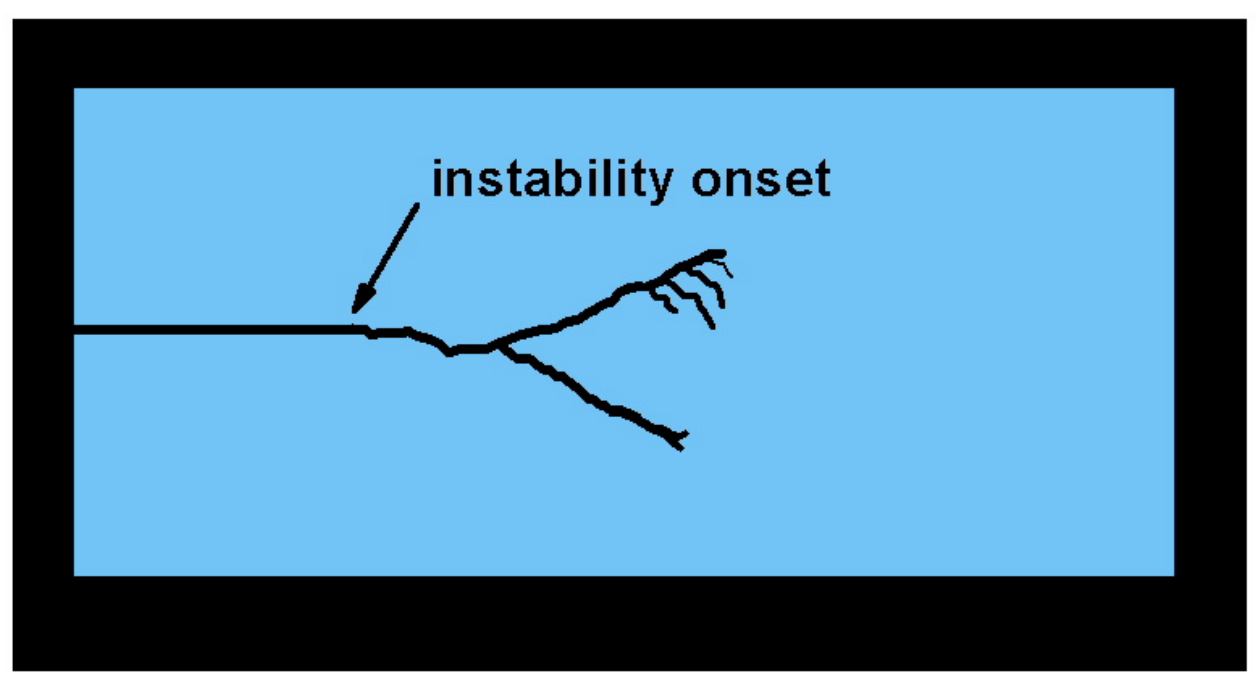


Figure 4

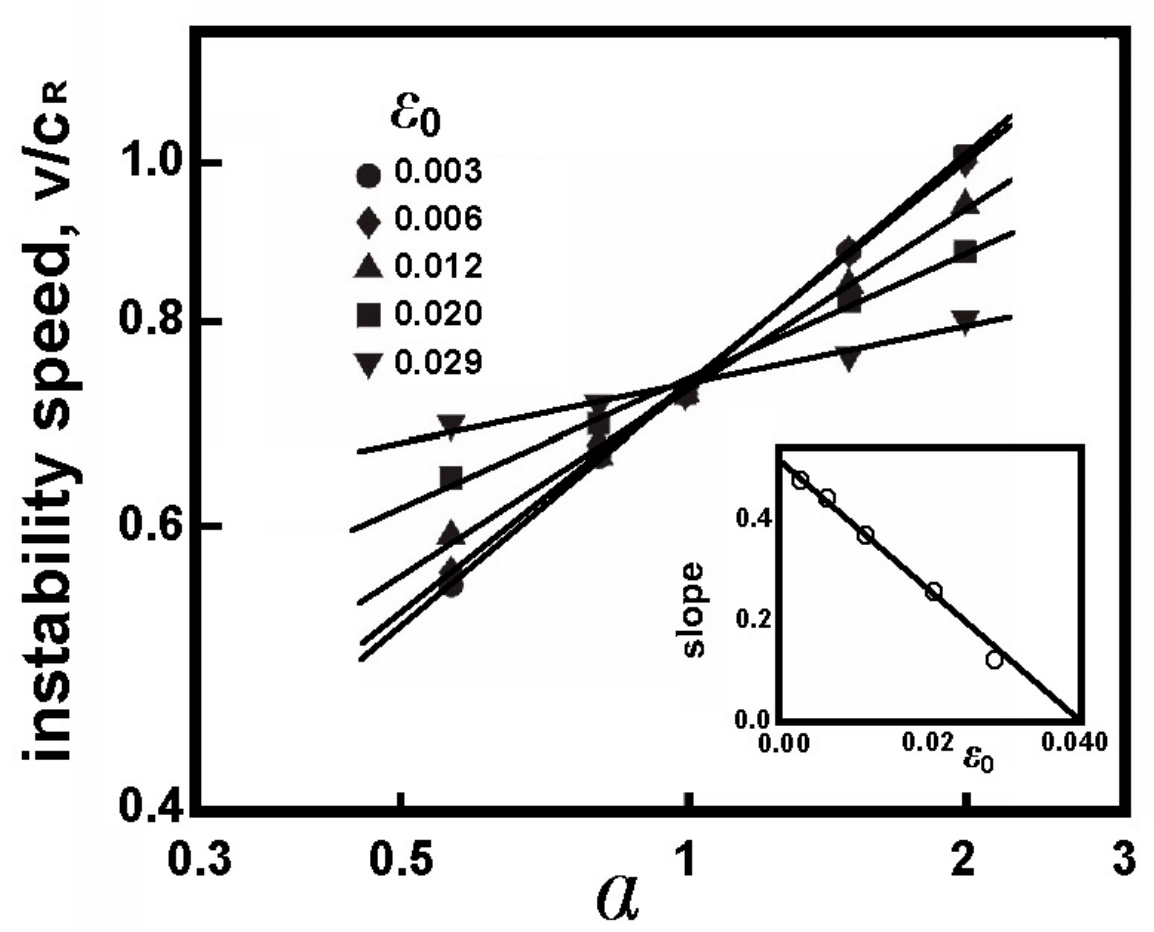


Figure 5

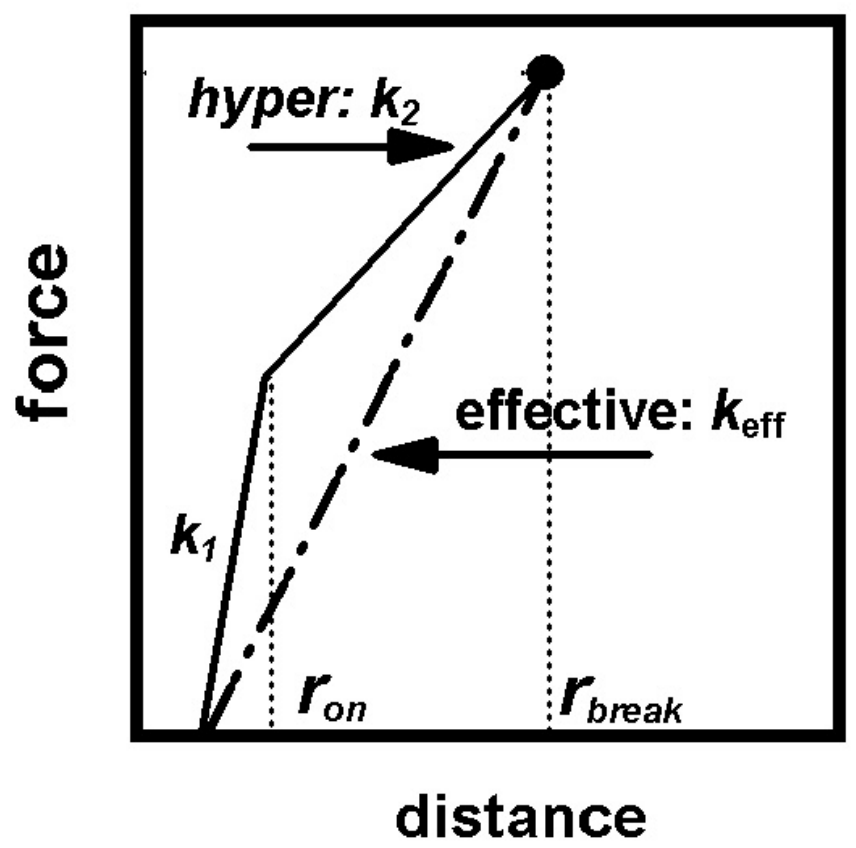


Figure 6

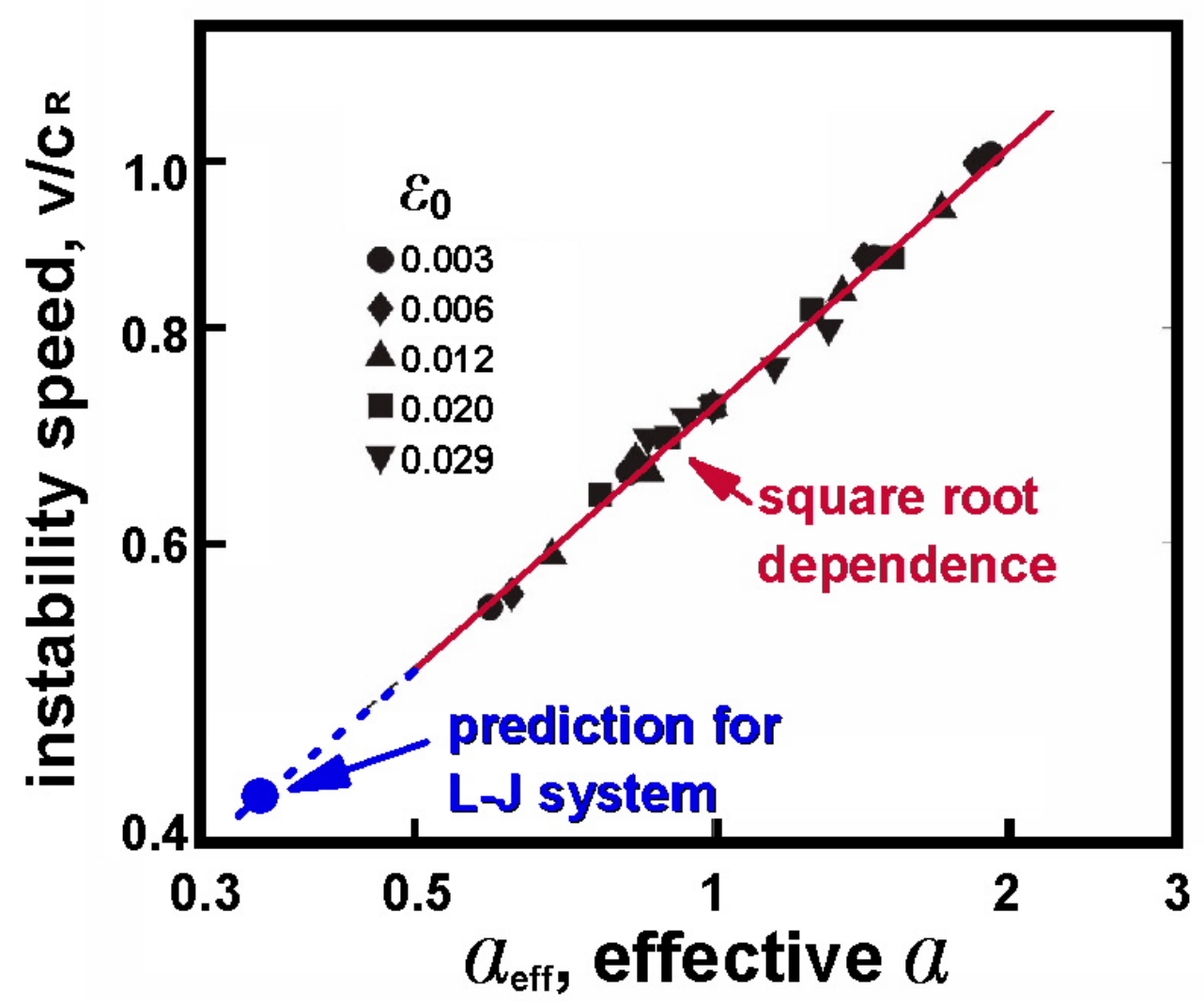


Figure 7

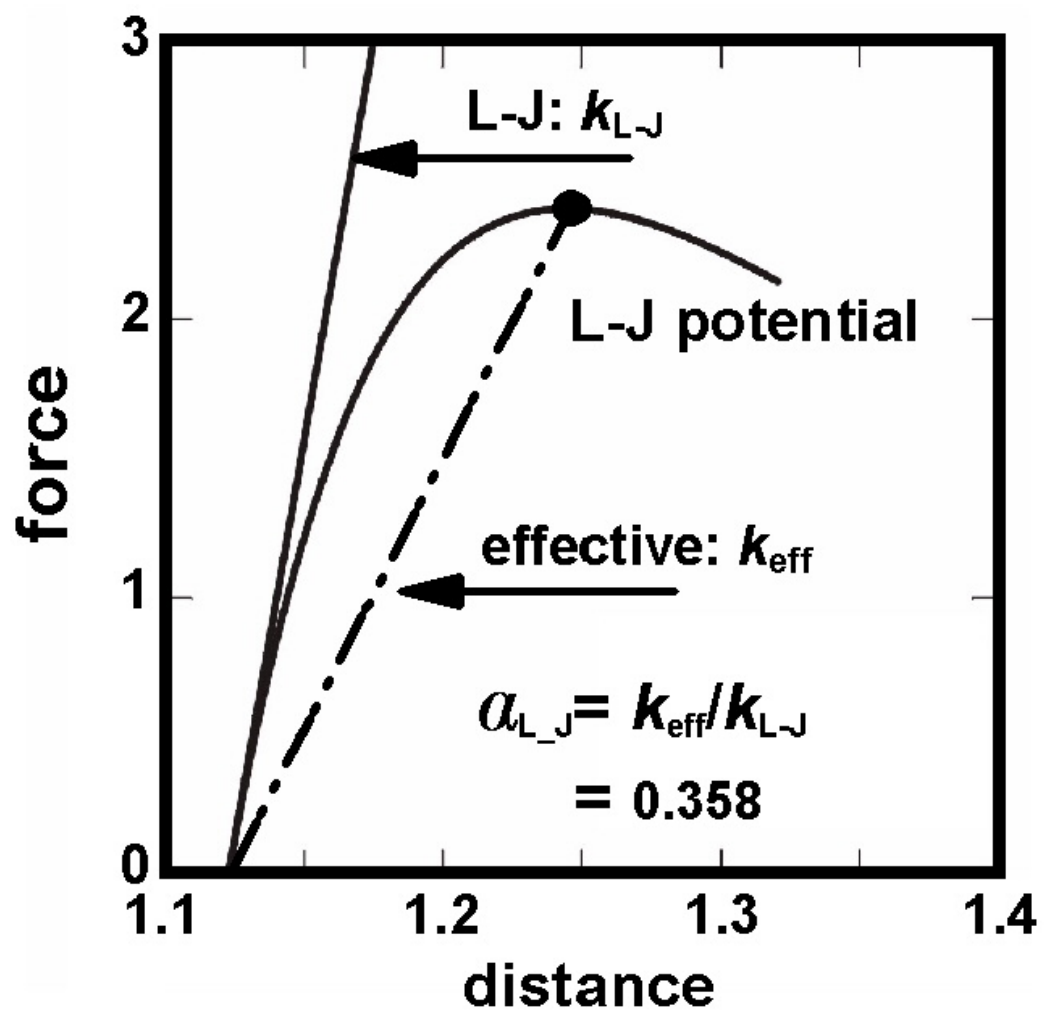


Figure 8
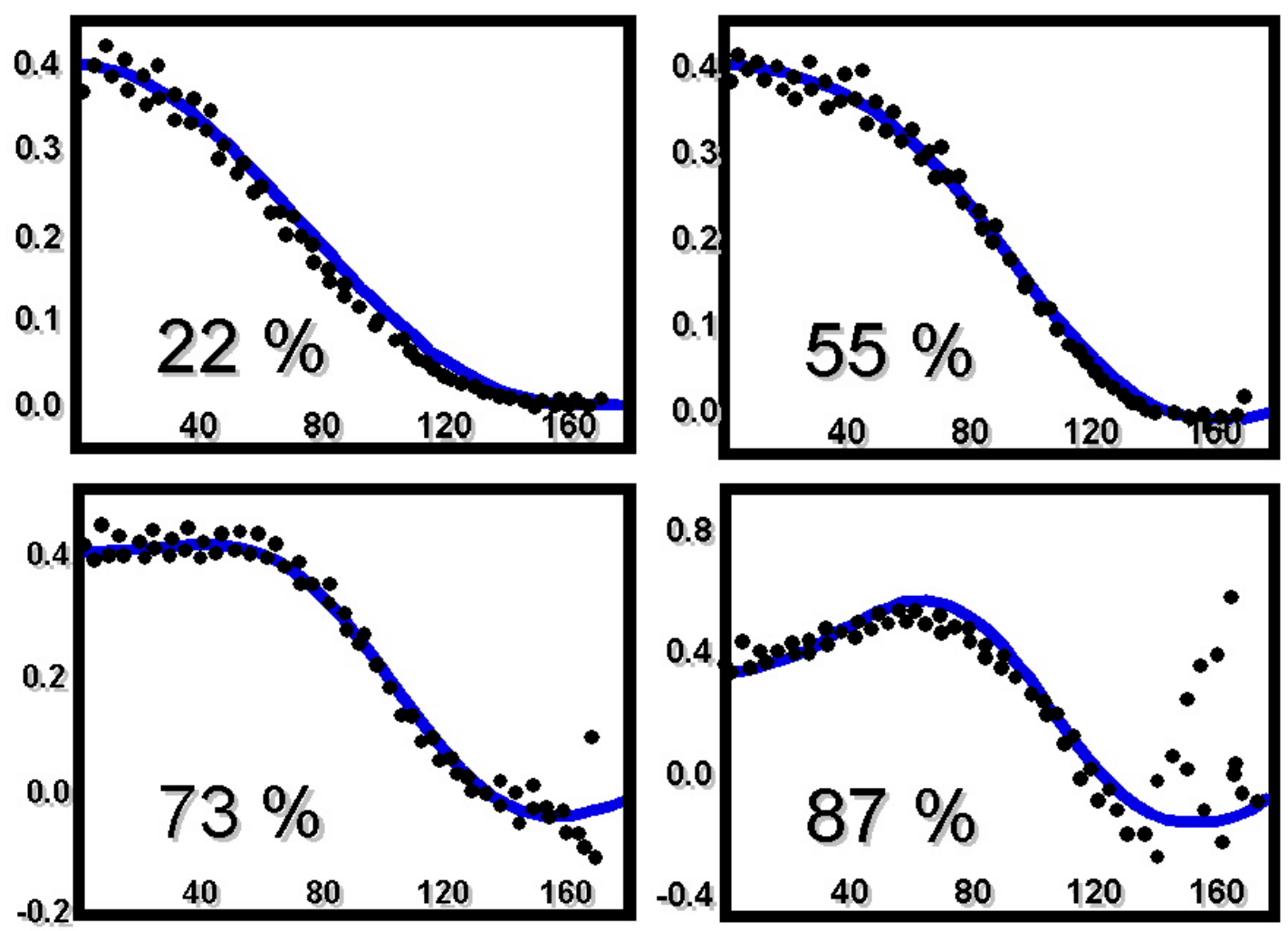